\title{
Pressure Fluctuation Characteristics of Narrow Gauge Train Running Through Tunnel*
}

\author{
Masahiro SUZUKI** and Yutaka SAKUMA** \\ **Railway Technical Research Institute, \\ 2-8-38 Hikari-cho, Kokubunji-shi, Tokyo 185-8540, JAPAN \\ E-mail: suzuki@rtri.or.jp
}

\begin{abstract}
Pressure fluctuations on the sides of narrow $(1067 \mathrm{~mm})$ gauge trains running in tunnels are measured for the first time to investigate the aerodynamic force acting on the trains. The present measurements are compared with earlier measurements obtained with the Shinkansen trains. The results are as follows: (1) The aerodynamic force, which stems from pressure fluctuations on the sides of cars, puts the energy into the vibration of the car body running through a tunnel. (2) While the pressure fluctuations appear only on one of the two sides of the trains running in double-track tunnels, the fluctuations in opposite phase on both sides in single-track tunnels. (3) The on-track test data of the narrow gauge trains show the same tendency as those of the Shinkansen trains, although it is suggested that the pressure fluctuations develop faster along the narrow gauge trains than the Shinkansen trains.
\end{abstract}

Key words: Narrow Gauge Train, Aerodynamic Force, Tunnel, Flow-induced Vibration, On-track Test

\section{Introduction}

As the maximum speed of Shinkansen trains in Japan has increased, lateral vibrations of the train in tunnels have become a subject of discussion from the viewpoint of ride comfort $^{(1)}$. As one of the factors causing the phenomenon, aerodynamic force has attracted attention. Full-scale measurements conducted on the various types of Shinkansen trains elucidated characteristics of the aerodynamic force as follows ${ }^{(2)}$ : (1) Continuous pressure fluctuation, which mainly causes the unsteady aerodynamic force, emerges on the side of train facing the tunnel wall. (2) The magnitude of the pressure fluctuation increases proportionately to the square of the airspeed relative to the train. (3) The pressure fluctuation increases from the head car to the $6 \sim 8$ th car, then remains constant, and finally increases drastically at the tail of the train set. The peak frequencies of the pressure fluctuation that are recognized after the $3 \mathrm{rd}$ car decrease from the $3 \mathrm{rd}$ car toward the $6 \sim 8$ th car and remains at the same level to the tail of the train set.

Numerical simulations clarified that the vortices arising around the bottom of the car body and their travel along the car side are the causes of the pressure fluctuation developing along the whole train set ${ }^{(2)}$. The study also revealed that the cause of the large pressure fluctuation at the train tail is the flow separation by the sudden expansion of the effective flow area.

Heretofore, a number of researches have been made over the decade on aerodynamic force acting on the Shinkansen trains running through the tunnels ${ }^{(2-5)}$. However, there is a potential of emerging the same phenomenon on the other high-speed trains (hereafter we call them 'narrow gauge train' since all of Japan Railways Group's network is narrow gauge of $1067 \mathrm{~mm}$ except for the Shinkansen and a few other lines with standard gauge of 1435

${ }^{*}$ Received 1 Mar., 2010 (No. Te-10-0104) [DOI: 10.1299/jmtl.3.469]

Copyright $($ C 2010 by JSME 
$\mathrm{mm}$ ). Because the magnitude of the pressure fluctuation increases proportionately to the square of the airspeed relative to the train as described above, and the airspeed relative to the narrow gauge train running inside a single-track tunnel, where its cross-sectional area ratio of the car body to the tunnel is large, may be comparable to that of the Shinkansen train in the double-track tunnel. However, the aerodynamic force acting on the narrow gauge trains have rarely been examined. Therefore, we conducted on-track tests on the narrow gauge trains. In this paper, we present results of those tests and compare them with those of the Shinkansen trains.

\section{On-track tests}

We conducted on-track tests on two types of narrow $(1067 \mathrm{~mm})$ gauge trains, namely Trains A and B in a similar way as we did on the Shinkansen trains ${ }^{(2)}$. Here, we describe its method briefly. We measured pressure and vibrating acceleration on 5 th car of a five-car train set of Train A, those on 5th car of a five-car train set of Train B and those on 9th car of a nine-car train set of Train B. Four pressure sensors were pasted on each side of the car. Accelerometers were mounted on the floor of the aisle just above each bogie to measure the lateral acceleration of the car body. Figure 1 demonstrates the experimental setup of the pressure sensors and the accelerometers installed on the train.

Figure 2 explains geometrical relations between the tunnel and the train. Train A runs inside a single-track tunnel, while Train B a double-track one. For reference purpose, a Shinkansen train and its double-track tunnel are also displayed.



(a) Side view

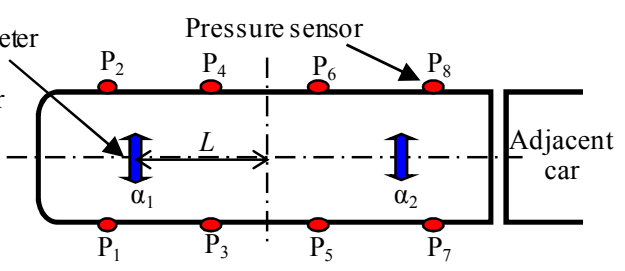

(b) Top view

Fig. 1 The experimental setup of pressure sensors and accelerometers

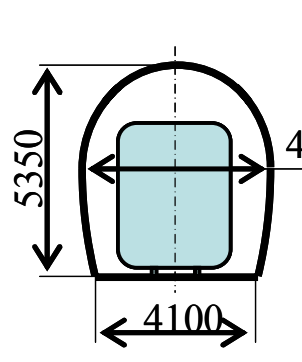

(a) A narrow gauge train in a single-track tunnel

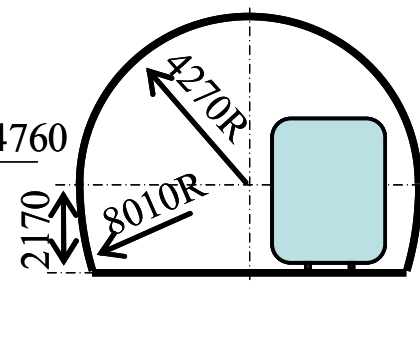

(b) A narrow gauge train in a double-track tunnel

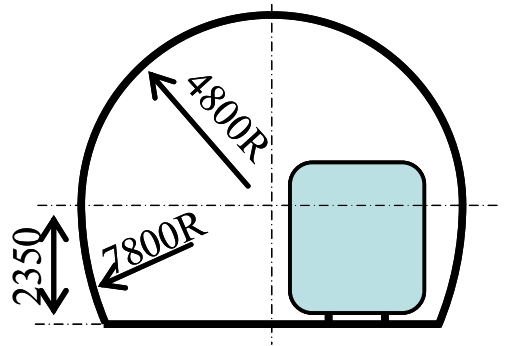

(c) A Shinkansen train in a double-track tunnel

Fig. 2 The cross sections of trains and tunnels (unit: $\mathrm{mm}$ )

\section{Results and discussion}

\subsection{Properties of the aerodynamic force acting on the narrow gauge trains}

First of all, with the results of the on-track test with Train A, we explain properties of the phenomenon. Figure 3 is a typical chart of the pressure and the vibrating acceleration of the tail car entering a single-track tunnel. First, a compression wave is generated when the front nose of the train set enters the tunnel. Second, an expansion wave is also occurred at the tail end of the train set entering the tunnel. There pressure waves travel 
toward the opposite exit of the tunnel at the speed of sound. Third, pressure reduces rapidly after the expansion wave passed the train. Fourth, the compression pressure waves are reflected at the distant tunnel exit and the resulted expansion wave encounters the train. During a period (indicated as sampling period in Fig. 3(a)) between an instant when the expansion wave passes the train and an instant when the train meets the reflected expansion wave, continuous pressure fluctuation emerges. While the larger pressure fluctuation appears only on the side facing the tunnel wall of the Shinkansen $\operatorname{train}^{(2)}$, the fluctuations in opposite phase on the both sides of the narrow gauge train in the single-track tunnel as shown in Fig. 3(c). (In the case of Train B running through a double-track tunnel, the large pressure fluctuation appears only on the car side facing the tunnel wall in the same way as the case of the Shinkansen train, however it is not shown here.) The difference in pressure between the both sides (hereafter referred to as pressure-difference) acts on the vehicle as aerodynamic force. As shown in Fig. 3(d), pressure-difference becomes larger after the train enters the tunnel. Figure 3(e) also shows that the amplitude of the vibrating acceleration increases in the tunnel section.

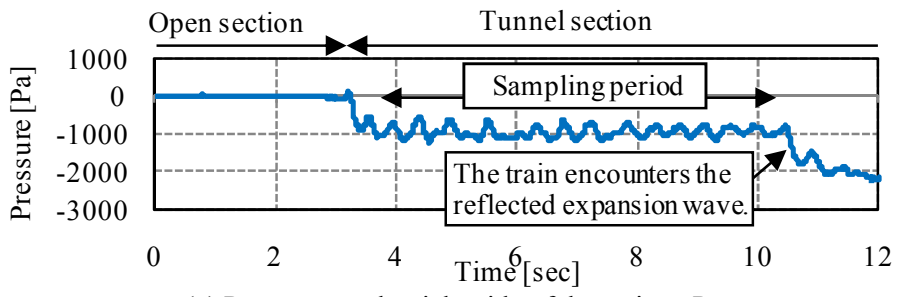

(a) Pressure on the right side of the train at $P_{1}$

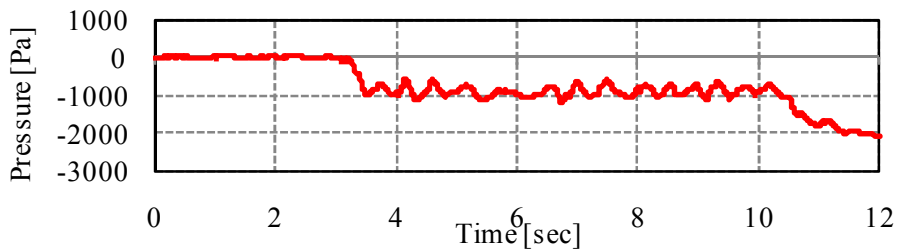

(b) Pressure on the left side of the train at $\mathrm{P}_{2}$

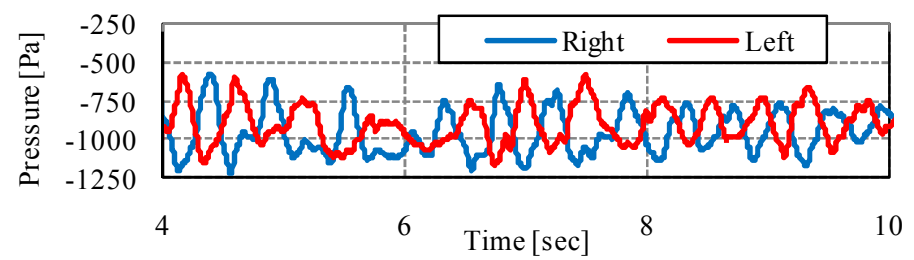

(c) Pressure on the both side of train during the sampling period (Enlarged view)

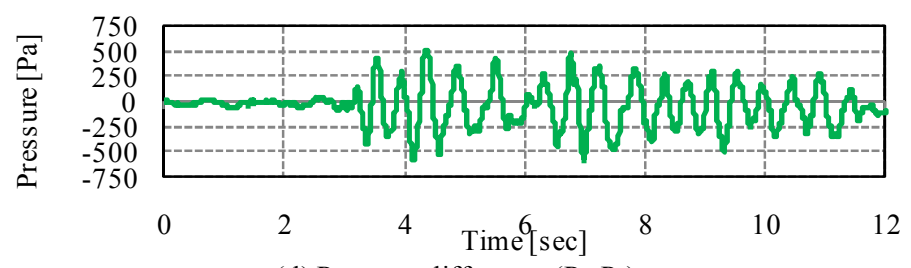

(d) Pressure difference $\left(\mathrm{P}_{1}-\mathrm{P}_{2}\right)$



(e) Vibrating accleration at $\alpha_{1}$

Fig. 3 Time histories of pressures and vibrating acceleration of the tail car of Train A entering a single-track tunnel (Train speed: $130 \mathrm{~km} / \mathrm{h}$ ) 
Since it is difficult to measure directly the aerodynamic forces acting on the running cars of on-track tests, those acing on Shinkansen trains are approximated based on the pressure data obtained at a limited number of measuring points ${ }^{(2)}$. In fact, a model experiment has verified that this approximation is applicable to the intermediate part of the train where local pressure fluctuations are relatively moderate ${ }^{(8)}$. Considering the fact that the nose of the narrow gauge train is short and the most of its body is intermediate part, we estimate the aerodynamic force in the same way used in Shinkansen trains: $\quad F=\sum_{i=1}^{4} \Delta p_{i} s_{i}$ and $M=\sum_{i=1}^{4} \Delta p_{i} s_{i} l_{i}$, where $F$ is the aerodynamic lateral force, $M$ the aerodynamic yawing moment, $\Delta p$ pressure-difference, $s_{i}$ the representative area of the $i$-th segment of the train side (grey area in Fig. 1), $l_{i}$ the moment arm of the $i$-th segment, which is the distance between the car center and the center of the segment (Fig. 1). Figure 4 demonstrates the root mean square values of the aerodynamic forces during the sampling period. The data on curve sections were excluded to delete the influence of the centrifugal force and the tilt of the cars due to the cant of tracks. Although the aerodynamic forces acting on the front car are small both in the open and the tunnel sections, those on the tail car are large in the tunnel section.

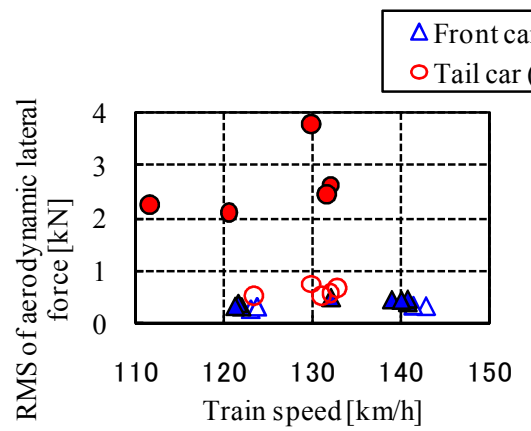

(a) Lateral force
$\Delta$ Front car (Tunnel section) - Tail car (Tunnel section)
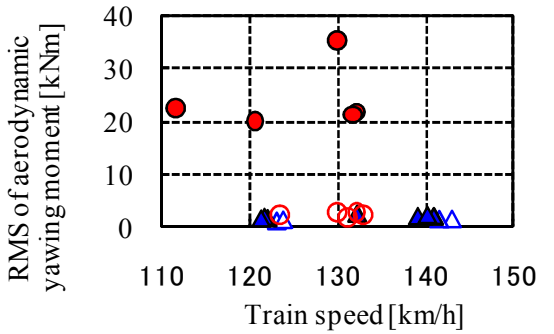

(b) Yawing moment

Fig. 4 The aerodynamic force acting on the front and tail cars of Train A running inside the single-track tunnel

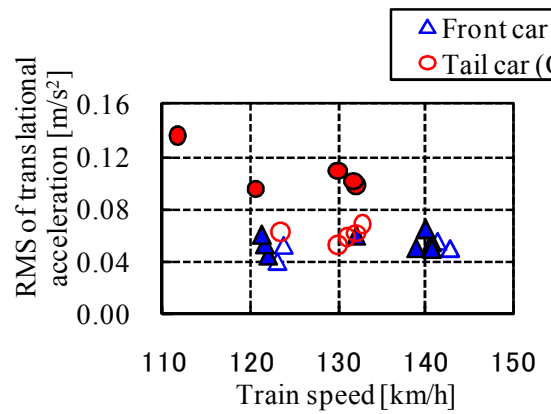

(a) Translational acceleration

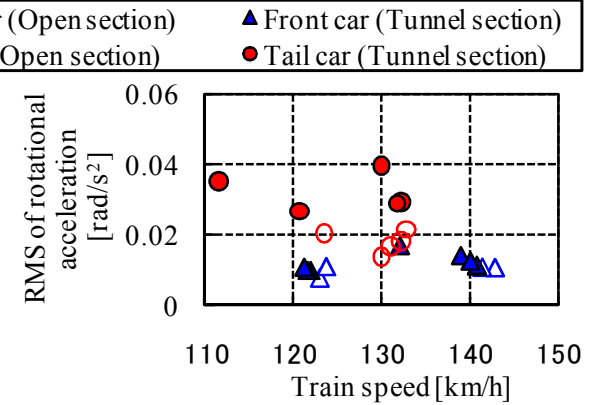

(b) Rotational acceleration

Fig. 5 The vibrating acceleration of the front and tail cars of Train A running inside the single-track tunnel

Figure 5 depicts the root mean square values of the vibrating accelerations. Here, translational acceleration is calculated by $\alpha_{t}=\left(\alpha_{1}+\alpha_{2}\right) / 2$ and rotational acceleration $\alpha_{t}=\left(\alpha_{1}-\alpha_{2}\right) / L$, where $\alpha_{1}$ and $\alpha_{2}$ are the lateral accelerations and $L$ the distance between the center of the car and that of the bogie (see Fig. 1). The values of the tail car running in the tunnel section are large. 
Figure 6 demonstrates typical time histories of the aerodynamic force and the vibrating acceleration of the tail car running inside the single-track tunnel. Good correlations between the aerodynamic lateral force and the translational acceleration and between the aerodynamic yawing moment and the rotational acceleration are observed, respectively.

The work done by the aerodynamic force on the car is calculated by integrating the product of the aerodynamic force and lateral velocity of the car, which is obtained by integrating the lateral acceleration. Figure 7 exhibits an example of the work done by the aerodynamic force on the tail car in the open section for a period of five seconds and in the tunnel section for a period of ten seconds, which includes the period of two seconds shown in Fig. 6. Because the open sections the train traveled contain few long straight tracks, the period for the open section is shorter than that for the tunnel. The train speed is $132 \mathrm{~km} / \mathrm{h}$ in both cases. While the aerodynamic force hardly work at all in the open section, it does in the tunnel section. Therefore the aerodynamic force puts the energy into the train vibration in the tunnel section.

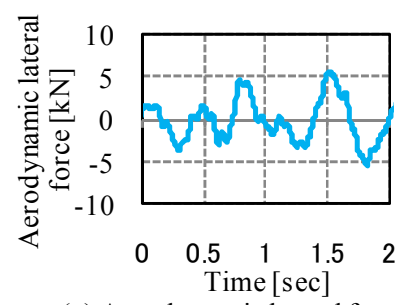

(a) Aerodynamic lateral force

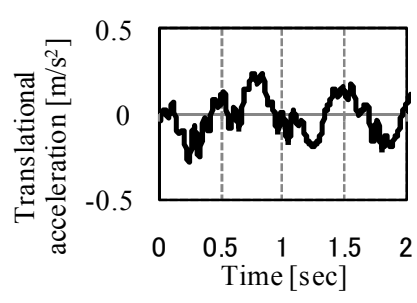

(c) Translational acceleration

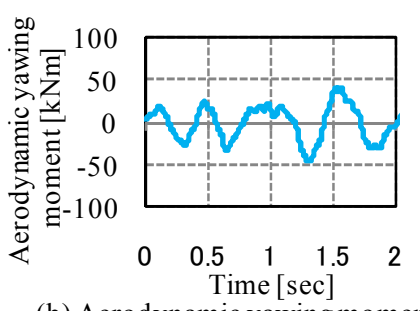

(b) Aerodynamic yawing moment

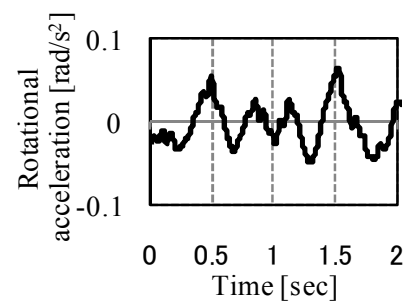

(d) Rotational acceleration

Fig. 6 Time histories of the aerodynamic force and the vibration of the tail car of Train A running inside the single-track tunnel (Train speed: $132 \mathrm{~km} / \mathrm{h}$ )

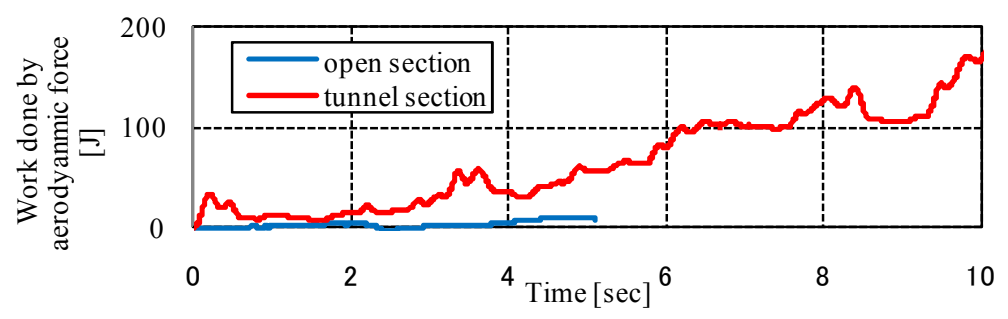

Fig. 7 An example of the work done by the aerodynamic force

(The tail car of Train A running inside the single-track tunnel. Train speed: $132 \mathrm{~km} / \mathrm{h}$ )

Next, comparing the results of Trains A and B, we discuss a relation between the train speed and the aerodynamic force acting the tail car in the tunnel section. Figure 8 shows a plot of the root mean square values of the aerodynamic force using the train speed on the horizontal axis. As the train speed increases, the aerodynamic force becomes larger. At the same train speed, the aerodynamic forces of Train A are larger than those of Train B. The reason is mainly due to the airspeed relative to the train, rather than the train shape. The airspeed relative to Train A running inside the single-track tunnel is larger than that to 
Train B running inside the double-track tunnel. The airspeed relative to the train can be calculated by the method of characteristics ${ }^{(6)}$. Using the airspeed relative to the train on the horizontal axis, the aerodynamic force is almost proportional to the square of the airspeed irrespective of the single-track tunnel or the double-track one as displayed in Fig. 9.

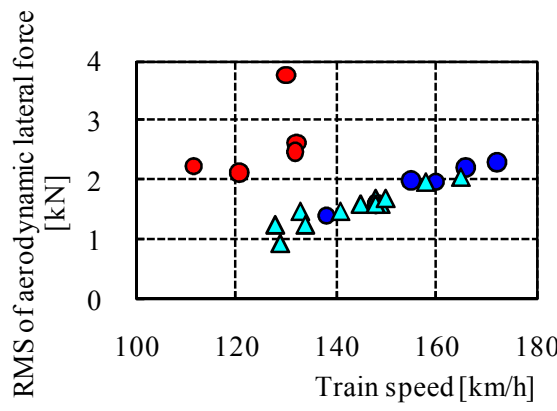

(a) Lateral force

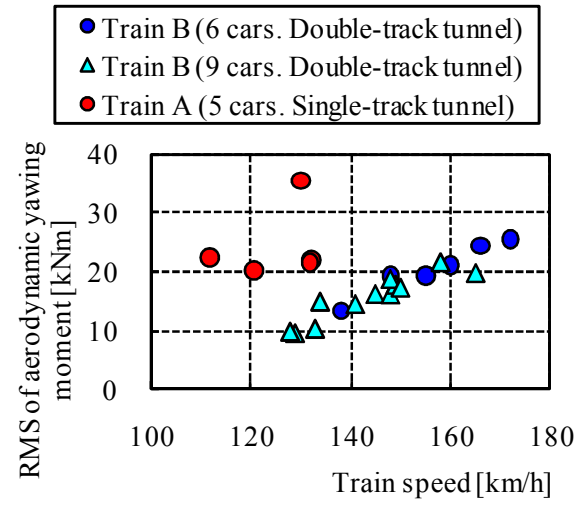

(b) Yawing moment

Fig. 8 The aerodynamic force acting on the tail car as a function of the train speed

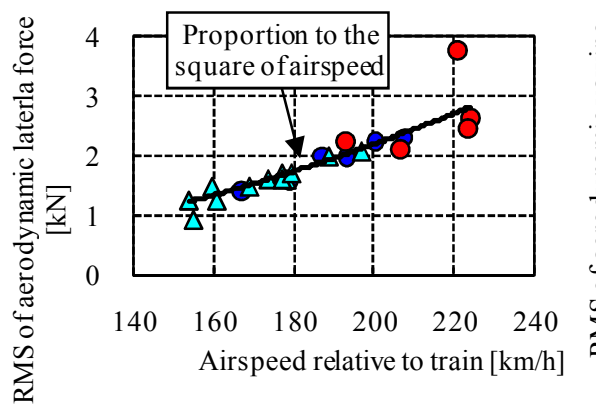

(a) Lateral force



(b) Yawing moment

Fig. 9 Aerodynamic force acting on the tail car as a function of the airspeed relative to the train

\subsection{Comparison with the Shinkansen trains}

From the above discussion, we have revealed that the pressure fluctuation emerges not only on the Shinkansen trains, but also on the narrow gauge trains. In this section, introducing non-dimensional parameters, we compare the present results with reported data of the Shinkansen trains ${ }^{(2)}$.

First, since the magnitude of pressure-difference fluctuation increases proportionately to the square of the airspeed relative to the train, we define a non-dimensional parameter which indicates the magnitude of pressure-difference fluctuation independent of the airspeed as follows:

Coefficient of pressure-difference fluctuation

$$
C_{\Delta p_{r m s}}=\frac{2(\Delta p)_{r m s}}{\rho u^{2}}
$$

where $(\Delta p)_{r m s}$ is the root mean square of pressure-difference $[\mathrm{Pa}], \quad \rho$ is the density of air $\left[\mathrm{kg} / \mathrm{m}^{3}\right], \quad u$ is the airspeed relative to the train $[\mathrm{m} / \mathrm{s}]$. 
Second, since it is reported that the peak frequencies of the pressure-difference are proportional to the airspeed relative to the train, we define a non-dimensional parameter which indicates the peak frequency of pressure-difference fluctuation independent of the airspeed as follows:

Non-dimensional peak frequency of pressure-difference fluctuation

$$
f_{\Delta p}^{\prime}=f_{\Delta p} \cdot \frac{d}{u}
$$

where $f_{\Delta p}$ is the peak frequency of pressure-difference fluctuation $[\mathrm{Hz}], d$ is a reference length. Since the previous experiment suggested that the peak frequency has a relation with the width of the car body ${ }^{(7)}$, we employ the car width as $d$.

Figure 10 plots the above two parameters obtained with the present data and the reported data of six types of the Shinkansen train. The horizontal axis indicates the distance from the head of the train set. In Fig. 10 (a), the values of Train A at $103 \mathrm{~m}$ and Train B at $124 \mathrm{~m}$ and $188 \mathrm{~m}$ (symbols encircled with the red line) are prominent as well as some of the Shinkansen trains (ones encircled with the blue line). These values are at the tail of the train set, where flow separates with large pressure fluctuation. Except for them, the values of the narrow gauge trains are almost same as those of the Shinkansen trains. Some of the values are slightly larger than those of the Shinkansen trains. This seems to be mainly due to the difference in smoothness of the body surface between the narrow gauge trains and the Shinkansen trains. As seen in Fig. 10 (b), the results of the narrow gauge trains exhibit the same tendency as the Shinkansen trains, in which the peak frequencies decrease along the train set. The peak frequencies of the narrow gauge trains decrease earlier than those of the Shinkansen trains. It is presumed that the roughness of the body, especially bottom parts, of the narrow gauge trains enhances the development of the pressure fluctuation and it results in the early decrease of the peak frequencies. Figure 10 (b) also indicates that the peak frequencies at the intermediate part of the train set are almost same as those at the tail in both cases of the Shinkansen trains and the narrow gauge ones. It is assumable that this is due to some interactions of flows between the intermediate part and the nose, however, its mechanism is unclear.

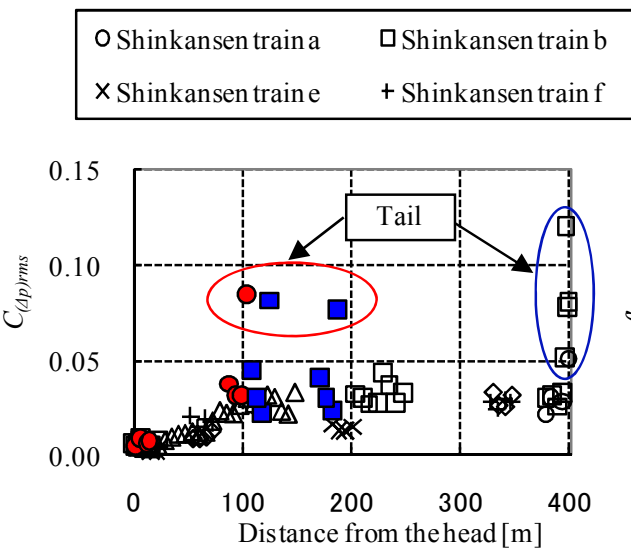

(a) Coefficient of pressure-difference fluctuation

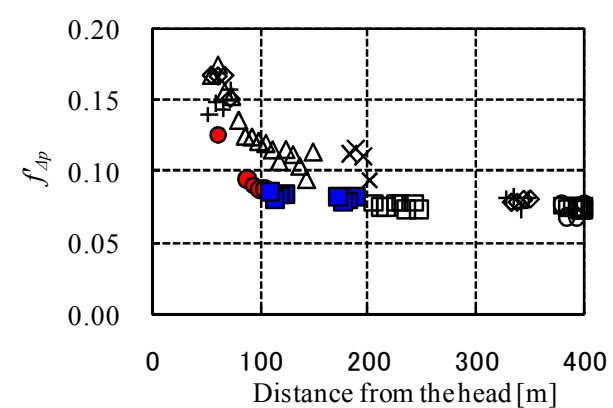

(b) Non-dimensional peak frequency of pressure-difference fluctuation

Fig. 10 Coefficient of pressure-difference fluctuation and non-dimensional peak frequency of pressure-difference fluctuation

\section{Conclusions}

We conducted the on-track tests on the narrow gauge trains to clarify the aerodynamic force acting on the cars running inside the tunnels. The measured data were compared 
with those of the Shinkansen trains. The results are as follows: (1) The aerodynamic force, which stems from pressure fluctuations on the sides of cars, puts the energy into the vibration of the car body running through a tunnel. (2) While the pressure fluctuations appear only on one of the two sides of trains travelling in double-track tunnels, the fluctuations in opposite phase on both sides in single-track tunnels. (3) The on-track test data of the narrow gauge trains show the same tendency as those of the Shinkansen trains, although it is suggested that the pressure fluctuations develop faster along the narrow gauge trains than the Shinkansen trains.

Although we assume that countermeasures ${ }^{(8)}$ for reducing the aerodynamic force acting on the Shinkansen trains can be also applied to the narrow gauge trains, countermeasures most suited to them are issues in the future.

\section{Acknowledgement}

The authors would like to thank West Japan Railway Co. and Hokkaido Railway Co., who have kindly cooperated with us on the on-track tests.

\section{References}

(1) Fujimoto, H., Miyamoto, M. and Shimamoto, Y., Lateral vibration of a Shinkansen and its decreasing measure, RTRI Report, Vol. 9, No. 1, (1995), pp. 19-24. (in Japanese)

(2) Suzuki, M., Ido, A., Sakuma, Y. and Kajiyama, H.: Full-scale measurement and numerical simulation of flow around high-speed train in tunnel, Journal of Mechanical Systems for Transportation and Logistics, Vol. 1, No. 3, (2008), pp. 281-292.

(3) Ishihara, T., Utsunomiya, M., Okumura, M., Sakuma, Y. and Shimomura, T., An investigation of lateral vibration caused by aerodynamic continuous force on high-speed train running within tunnels, Proceedings of World Congress on Railway Research, Vol. E, (1997), pp. 531-538

(4) Nikko, S., Tanifuji, K., Sakanoue, K. and Nanba, K., Modeling of aerodynamic force acting in tunnel for analysis of riding comfort in a train, Journal of Mechanical Systems for Transportation and Logistics, Vol. 1, No. 1, (2008), pp. 31-42.

(5) Diedrichs, B., Berg M., Stichel, S. and Krajnović S., Vehicle dynamics of a high-speed passenger car due to aerodynamics inside tunnels, Proceedings of the Institution of Mechanical Engineers, Part F: Journal of Rail and Rapid Transit, Vol. 221, (2007), pp. 527-545.

(6) Iida, M. and Maeda, T., Numerical calculation of pressure transients in railway tunnels, RTRI Report, Vol. 4, No. 7, (1990), pp. 54-62. (in Japanese)

(7) Suzuki, M., Studies of the flow fields around a train in tunnel, Proceedings of Conference on Modeling Fluid Flow, (2003), pp. 702-703.

(8) Suzuki, M., Nakade K. and Ido, A., Countermeasures for reducing unsteady aerodynamic force acting on high-speed train in tunnel by use of modifications of train shapes, Journal of Mechanical Systems for Transportation and Logistics, Vol. 2, No. 1, (2009), pp. 1-12. 